\title{
Spatial inhomogeneity of magnetic moments in the cobalt oxide spinel $\mathrm{Co}_{3} \mathrm{O}_{4}$
}

\author{
Y. Ikedo, ${ }^{1}$ J. Sugiyama,${ }^{1}$ H. Nozaki, ${ }^{1}$ H. Itahara,${ }^{1}$ J. H. Brewer, ${ }^{2}$ E. J. Ansaldo,${ }^{3}$ G. D. Morris,${ }^{3}$ \\ D. Andreica, ${ }^{4}$ and A. Amato ${ }^{4}$ \\ ${ }^{1}$ Toyota Central Research and Development Laboratories, Inc., Nagakute, Aich, 480-1192 Japan \\ ${ }^{2}$ TRIUMF, CIAR, and Department of Physics and Astronomy, University of British Columbia, Vancouver, BC, V6T IZ1 Canada \\ ${ }^{3}$ TRIUMF, 4004 Wesbrook Mall, Vancouver, BC, V6T $2 A 3$ Canada \\ ${ }^{4}$ Laboratory for Muon-Spin Spectroscopy, Paul Scherrer Institut, Villigen PSI, Switzerland \\ (Received 31 May 2006; revised manuscript received 8 December 2006; published 28 February 2007)
}

\begin{abstract}
The antiferromagnetic (AF) nature of the normal spinel $\mathrm{Co}_{3} \mathrm{O}_{4}$ with Néel temperature $\left(T_{\mathrm{N}}\right)=30 \mathrm{~K}$ was investigated by means of positive muon spin rotation and relaxation $\left(\mu^{+} \mathrm{SR}\right)$ techniques using a polycrystalline sample. Clear muon spin precession signals due to a quasistatic long-range AF order were found in the zero-field $\mu^{+} \mathrm{SR}$ spectra below $T_{\mathrm{N}}$. The spectra consist of two oscillating signals with frequencies at $T \rightarrow 0 \mathrm{~K}$ of 80 and $60 \mathrm{MHz}$, respectively, indicating an incommensurate (IC) $\mathrm{AF}$ order in $\mathrm{Co}_{3} \mathrm{O}_{4}$. A possible reason for the appearance of the IC-AF order in $\mathrm{Co}_{3} \mathrm{O}_{4}$ would be local structural transitions due to a charge and/or a spin state change of Co ions.
\end{abstract}

DOI: 10.1103/PhysRevB.75.054424

PACS number(s): 76.75.+i, 75.25.+z, 75.50.Ee

\section{INTRODUCTION}

The magnetic properties of the cobalt oxide spinel $\left(\mathrm{Co}_{3} \mathrm{O}_{4}\right)$ have been extensively studied previously by ${ }^{59} \mathrm{Co}$ nuclear magnetic resonance $\left({ }^{59} \mathrm{Co}-\mathrm{NMR}\right),{ }^{1,2}$ magnetic susceptibility $(\chi),{ }^{3}$ neutron diffraction, ${ }^{4}$ Mössbauer effect, ${ }^{5}$ and heat capacity ${ }^{6}\left(C_{\mathrm{p}}\right)$ measurements, because of its unique magnetic structure and relatively strong superexchange interaction, which arises in spite of the large distance $(3.89 \AA)$ between magnetic Co ions. ${ }^{4}$ In the last decade, both superparamagnetism and ferrimagnetism in $\mathrm{Co}_{3} \mathrm{O}_{4}$ nanoparticles have also attracted much attention not only for their fundamental interest but also for their potential applications in the realm of nanotechnology. ${ }^{7-9}$

The crystal structure of $\mathrm{Co}_{3} \mathrm{O}_{4}$ is a cubic normal spinel with a space group $O_{h}^{7}-F d \overline{3} m$, lattice constant $a=8.084 \AA$, and oxygen parameter $u=0.392$ at ambient temperature. ${ }^{10}$ The charge distribution for $\mathrm{Co}_{3} \mathrm{O}_{4}$ is represented by $\left[\mathrm{Co}^{2+}\right]_{8 a}\left[\mathrm{Co}_{2}^{3+}\right]_{16 d}\left[\mathrm{O}_{4}^{2-}\right]_{32 e}$, in which $8 a$ denotes the tetrahedral site and $16 d$ the octahedral site surrounded by $\mathrm{O}^{2-}$ ions at $32 e$ sites (see Fig. 1). Past work showed that $\mathrm{Co}^{3+}$ ions at the $16 d$ site are in a diamagnetic $t_{2 g}^{6}$ [low-spin (LS), $S=0$ ] state due to a strong octahedral cubic field and consequent large crystal-field splitting between $t_{2 g}$ and $e_{g}$ levels in the $3 d$ orbitals, while $\mathrm{Co}^{2+}$ ions at the $8 a$ site are in a high-spin (HS) $e_{g}^{4} t_{2 g}^{3}$ state with $S=3 / 2 .{ }^{4}$ It is also well known that $\mathrm{Co}_{3} \mathrm{O}_{4}$ undergoes a magnetic transition from a high- $T$ paramagnetic state to a low- $T$ long-range-ordered antiferromagnetic (AF) state at $T_{\mathrm{N}}=30 \mathrm{~K}$.

In the $\mathrm{Co}_{3} \mathrm{O}_{4}$ spinel lattice, $\mathrm{Co}^{2+}$ ions at the $8 a$ site form a diamond lattice, which consists of two displaced facecentered-cubic (fcc) sublattices with origins at $(0,0,0)$ and at $\left(\frac{1}{4}, \frac{1}{4}, \frac{1}{4}\right)$. The $\mathrm{Co}^{2+}$ ion at $(0,0,0)$ is thus tetrahedrally surrounded by four nearest-neighboring $(\mathrm{NN}) \mathrm{Co}^{2+}$ ions. Since the four $\mathrm{NN} \mathrm{Co}^{2+}$ ions form a fcc sublattice and the four $\mathrm{Co}^{2+}$ spins align antiparallel to the $\mathrm{Co}^{2+}$ spin at $(0,0,0)$, which belongs to the other fcc sublattice, the spin arrangement in each sublattice is ferromagnetic (FM) but it is $\mathrm{AF}$ between adjacent sublattices. ${ }^{4}$ As a result, the magnetic space group of $\mathrm{Co}_{3} \mathrm{O}_{4}$ changes from high- $T O_{h}^{7}-F d \overline{3} m$ to low- $T$ $T_{d^{-}}^{2} F \overline{4} 3 m$ at $T_{\mathrm{N}}$, although no structural phase transition was found down to the lowest $T$ measured. ${ }^{4}$ In this paper, we use site notations for $F d \overline{3} m$ even in the $\mathrm{AF}$ state for simplicity.

One of our motivations for investigating the magnetism of $\mathrm{Co}_{3} \mathrm{O}_{4}$ is the fact that $\mathrm{Co}_{3} \mathrm{O}_{4}$ is the parent compound for layered cobalt oxides such as $\mathrm{Na}_{x} \mathrm{CoO}_{2}$ (Ref. 11), $\left[\mathrm{Ca}_{2} \mathrm{CoO}_{3}\right]_{0.62}^{\mathrm{RS}}\left[\mathrm{CoO}_{2}\right]$ (Ref. 12), and $\left[\mathrm{Ca}_{2} \mathrm{Co}_{4 / 3} \mathrm{Cu}_{2 / 3} \mathrm{O}_{4}\right]_{0.62}^{\mathrm{RS}}\left[\mathrm{CoO}_{2}\right]$ (Ref. 13) (RS denotes a rocksalt-type sublattice), which exhibit metallic conductivity and an extraordinarily large thermopower. These features make them promising candidates as the basic material for thermoelectric power generation systems. In order to understand the origin of good thermoelectric performance in these layered cobalt oxides, we previously carried out positive muon spin rotation and relaxation $\left(\mu^{+} \mathrm{SR}\right)$ experiments on these materials, ${ }^{14-22}$ and found that a short-range incommensurate spin-density-wave (IC-SDW) order appears below $100 \mathrm{~K}\left(=T_{\mathrm{SDW}}^{\mathrm{on}}\right)$ and a long-range order forms below $30 \mathrm{~K}$ $\left(=T_{\mathrm{SDW}}^{\mathrm{end}}\right)$ for $\left[\mathrm{Ca}_{2} \mathrm{CoO}_{3}\right]_{0.62}^{\mathrm{RS}}\left[\mathrm{CoO}_{2}\right],{ }^{18}$ and $T_{\mathrm{SDW}}^{\mathrm{on}}=200 \mathrm{~K}$ and $T_{\mathrm{SDW}}^{\mathrm{end}}=140 \mathrm{~K}$ for $\left[\mathrm{Ca}_{2} \mathrm{Co}_{4 / 3} \mathrm{Cu}_{2 / 3} \mathrm{O}_{4}\right]_{0.62}^{\mathrm{RS}}\left(\mathrm{CoO}_{2}\right){ }^{20}$ Since a long-range commensurate $\mathrm{AF}$ order is also observed for

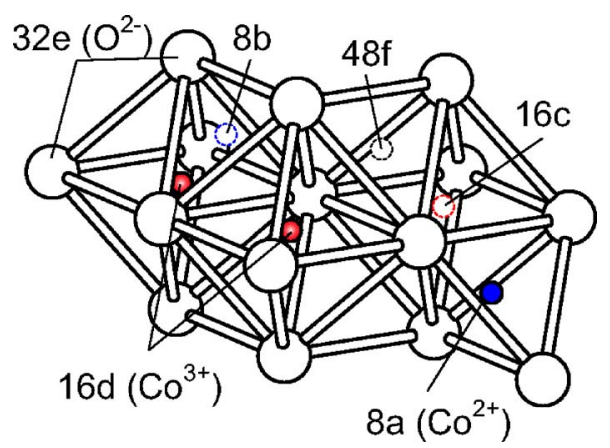

FIG. 1. (Color online) The crystal structure of $\mathrm{Co}_{3} \mathrm{O}_{4} \cdot 8 a$ (tetrahedron) and $16 d$ (octahedron) sites are occupied by $\mathrm{Co}^{2+}$ and $\mathrm{Co}^{3+}$ ions, respectively. $8 b$ and $48 f$ sites are empty tetrahedra and the $16 c$ site is empty octahedron. 
$\mathrm{Na}_{0.75} \mathrm{CoO}_{2},{ }^{15}$ we proposed that the IC-SDW lies not in the rocksalt-type $\left[\mathrm{Ca}_{2} \mathrm{CoO}_{3}\right]$ and/or $\left[\mathrm{Ca}_{2} \mathrm{Co}_{4 / 3} \mathrm{Cu}_{2 / 3} \mathrm{O}_{4}\right]$ sublattice but in the $\mathrm{CoO}_{2}$ planes.

More recent $\mu^{+} \mathrm{SR}$ and neutron scattering results on improved single-crystal samples, however, indicate that the magnetic ordering is more complex than postulated above, because of the following.

(i) The magnetic order for $\mathrm{Na}_{x} \mathrm{CoO}_{2}$ with $x=0.75$ and 0.82 was found to be $A$-type $\mathrm{AF}$-i.e., $\mathrm{FM}$ in the $\mathrm{CoO}_{2}$ plane but AF between adjacent $\mathrm{CoO}_{2}$ planes, by recent neutron experiments. ${ }^{23,24}$

(ii) The internal magnetic field at $T \rightarrow 0 \mathrm{~K}\left(H_{\text {int, }, 0 \mathrm{~K}}\right)$ corresponds to a muon spin precession frequency of $54 \mathrm{MHz}$ for $\left[\mathrm{Ca}_{2} \mathrm{CoO}_{3}\right]_{0.62}^{\mathrm{RS}}\left[\mathrm{CoO}_{2}\right]$ (Ref. 18) and $60 \mathrm{MHz}$ for $\left[\mathrm{Ca}_{2} \mathrm{Co}_{4 / 3} \mathrm{Cu}_{2 / 3} \mathrm{O}_{4}\right]_{0.62}^{\mathrm{RS}}\left[\mathrm{CoO}_{2}\right],{ }^{20}$ whereas it is $8 \mathrm{MHz}$ for $\mathrm{LiCoO}_{2},{ }^{22} \leqslant 3 \mathrm{MHz}$ for $\mathrm{Na}_{x} \mathrm{CoO}_{2},{ }^{15}$ and $<1.5 \mathrm{MHz}$ for $\mathrm{K}_{0.49} \mathrm{CoO}_{2} \cdot{ }^{21}$

(iii) The layered cobalt oxides $\left[\mathrm{Sr}_{2-x} \mathrm{~Pb}_{x} \mathrm{Bi}_{2} \mathrm{O}_{4}\right]_{0.5}\left[\mathrm{CoO}_{2}\right]$, which lack $\mathrm{Co}$ ions in the rocksalt-type sublattice, do not exhibit IC-SDW order but only a static Kubo-Toyabe muon spin relaxation below $\sim 3 \mathrm{~K}$, while the resistivity- $T$ curves show a broad minimum around $60 \mathrm{~K}$, which was thought to indicate the appearance of the IC-SDW order in that system. ${ }^{25}$

(iv) According to other past $\mu^{+} \mathrm{SR}$ work, $\nu\left(H_{\text {int }}\right)$ $\sim 55 \mathrm{MHz}$ for rocksalt $\mathrm{CoO}\left(T_{\mathrm{N}}=290 \mathrm{~K}\right){ }^{26}$

Since implanted muons in oxides bind preferentially with $\mathrm{O}^{2-}$ ions to form a $\mathrm{O}-\mu^{+}$bond (similar to the $\mathrm{O}-\mathrm{H}^{+}$hydrogen bond), the magnitude of $H_{\text {int }}$ detected by $\mu^{+} \mathrm{SR}$ is determined by both the distance (bond length) between $\mathrm{Co}$ and $\mathrm{O}$ ions $\left(d_{\mathrm{Co}-\mathrm{O}}\right)$ and the magnitude of the Co spin-i.e., moment. In $\left[\mathrm{Ca}_{2} \mathrm{CoO}_{3}\right]_{0.62}^{\mathrm{RS}}\left[\mathrm{CoO}_{2}\right], d_{\mathrm{Co}-\mathrm{O}}$ was reported to range from 1.8 to $2.0 \AA$ in the $\left[\mathrm{CoO}_{2}\right]$ sublattice and from 1.6 to $2.7 \AA$ in the $\left[\mathrm{Ca}_{2} \mathrm{CoO}_{3}\right]$ sublattice. ${ }^{27}$ The large variation in $d_{\mathrm{Co}-\mathrm{O}}$ in $\left[\mathrm{Ca}_{2} \mathrm{CoO}_{3}\right]$ is caused by a misfit between the two sublattices. Although the charge distribution of $\mathrm{Co}$ ions in $\left[\mathrm{Ca}_{2} \mathrm{CoO}_{3}\right]_{0.62}^{\mathrm{RS}}\left[\mathrm{CoO}_{2}\right]$ and/or $\left[\mathrm{Ca}_{2} \mathrm{Co}_{4 / 3} \mathrm{Cu}_{2 / 3} \mathrm{O}_{4}\right]_{0.62}^{\mathrm{RS}}\left[\mathrm{CoO}_{2}\right]$ is still unknown, the longer $d_{\mathrm{Co}-\mathrm{O}}$ in $\left[\mathrm{Ca}_{2} \mathrm{CoO}_{3}\right]$ than in $\left[\mathrm{CoO}_{2}\right]$ and common transport properties of the layered cobalt oxides suggest that both $\mathrm{Co}^{3+}$ and $\mathrm{Co}^{4+}$ coexist in $\left[\mathrm{CoO}_{2}\right]$, whereas the average valence is almost $3+$ for Co ions in the RS sublattice.

In the $\mathrm{Co}_{3} \mathrm{O}_{4}$ spinel, on the other hand, $d_{\mathrm{Co}^{3+}-\mathrm{O}}$ is $\sim 1.92 \AA$ and $d_{\mathrm{Co}^{2+}-\mathrm{O}} \sim 1.93 \AA$. The spinel $\mathrm{Co}_{3} \mathrm{O}_{4}$ is considered to be a cation-deficient rocksalt; that is, $\mathrm{O}^{2-}$ ions at the $32 e$ site and $\mathrm{Co}^{3+}$ ions at the $16 d$ site form an RS lattice together with the vacant $16 c$ site, while $\mathrm{Co}^{2+}$ ions locate at the interstitial $8 a$ site. By comparison with the layered cobalt oxides, $\mathrm{Co}_{3} \mathrm{O}_{4}$ possesses a simple structure with a well-known charge distribution in the lattice. We therefore initiated the present study of the magnetic properties of $\mathrm{Co}_{3} \mathrm{O}_{4}$ in order to obtain more insights into the nature of the layered cobalt oxides.

Furthermore, $\mu^{+} S R$, as it is very sensitive to the local magnetic environment, ${ }^{26,28,29}$ provides unique information on spatially inhomogeneous long-range ordering, such as an ICspin-density-wave (SDW) state. ${ }^{30,31}$ This is because the local magnetic field at a crystallographic $\mu^{+}$site varies depending on the spatial inhomogeneity, naturally generating a wide field distribution and as a result a rapidly relaxing oscillation.
In model AF cases, on the other hand, a long-lived oscillating signal is normally observed; i.e., the relaxation rate is very small, because the local magnetic field is in principle identical for each $\mu^{+}$site. In this paper, we report on both weak (relative to the spontaneous internal fields in the ordered state) transverse-field (wTF-) $\mu^{+} \mathrm{SR}$ and zero-field (ZF-) $\mu^{+} \mathrm{SR}$ for a polycrystalline $\mathrm{Co}_{3} \mathrm{O}_{4}$ sample at temperatures between 1.8 and $60 \mathrm{~K}$, with the aim of elucidating the nature of the AF phase of $\mathrm{Co}_{3} \mathrm{O}_{4}$ with $T_{\mathrm{N}}=30 \mathrm{~K}$.

\section{EXPERIMENT}

A polycrystalline sample of $\mathrm{Co}_{3} \mathrm{O}_{4}$ was synthesized by a tape-casting method using $\beta$ - $\mathrm{Co}(\mathrm{OH})_{2}$ crystal platelets as a starting material. The sample was sintered at $1200 \mathrm{~K}$ for $2 \mathrm{~h}$ in an $\mathrm{O}_{2}$ atmosphere followed by annealing in an $\mathrm{O}_{2}$ gas flow. The spinel structure of the sample was confirmed by x-ray diffraction measurements. The diffraction pattern showed only peaks due to $\mathrm{Co}_{3} \mathrm{O}_{4}$ spinel structure.

Magnetic susceptibility was measured using a superconducting quantum interference device (SQUID) magnetometer (MPMS, Quantum Design) at temperatures between 400 and $5 \mathrm{~K}$. Heat capacity was measured using relaxation technique (PPMS, Quantum Design) in the temperature range between 300 and $1.9 \mathrm{~K}$.

The $\mu^{+} S R$ experiments were performed on the surface muon beamline of M20 at TRIUMF and the $\pi \mathrm{E} 1$ beamline at PSI using the instrument Dolly. Experimental setup and techniques are described elsewhere. ${ }^{32}$

\section{RESULTS}

\section{A. Magnetic susceptibility and specific heat}

The magnetic susceptibility $\chi$ of the polycrystalline $\mathrm{Co}_{3} \mathrm{O}_{4}$ sample was measured in field cooling mode with $10 \mathrm{kOe}$ at temperatures from 400 to $5 \mathrm{~K}$. Figure 2 shows the temperature dependence of (a) $\chi$ and (b) $\chi^{-1}$. The inset of Fig. 2(a) shows the slope of $\chi(d \chi / d T)$ as a function of $T$. The $\chi(T)$ curve exhibits a broad maximum at around $40 \mathrm{~K}$, whereas the $d \chi / d T$ shows a sharp peak at $29 \mathrm{~K}$. Above $40 \mathrm{~K}, \chi$ decreases monotonically with increasing $T$, while $\chi^{-1}$ increases almost linearly with increasing $T$, indicating a Curie-Weiss paramagnetic behavior. Indeed, the $T$ dependence of $\chi$ above $100 \mathrm{~K}$ is well represented by

$$
\chi(T)=\chi_{0}+C /\left(T-\Theta_{\mathrm{p}}\right),
$$

where $\chi_{0}$ is the temperature-independent susceptibility, $C$ is the Curie-Weiss constant, and $\Theta_{\mathrm{p}}$ is the paramagnetic Curie temperature. The fit for the $\chi(T)$ curve using Eq. (1) over the $T$ range above $100 \mathrm{~K}$ yields $\Theta_{\mathrm{p}}=95 \pm 2 \mathrm{~K}, \chi_{0}=(1.86 \pm 0.07)$ $\times 10^{-3} \mathrm{emu} /\left(\mathrm{mol} \mathrm{Co}{ }^{2+}\right), \quad$ and $C=2.86 \pm 0.04 \mathrm{~K} \mathrm{emu} /$ $\left(\mathrm{mol} \mathrm{Co}^{2+}\right)$. The effective magnetic moment per mol Co $\left(\mu_{\text {eff }}\right)$ is calculated by using the formula $C=N \mu_{\text {eff }}^{2} / 3 k_{\mathrm{B}}$, where $N$ is the number density of $\mathrm{Co}^{2+}$ ions per mol and $k_{\mathrm{B}}$ is the Boltzmann constant. We obtain $\mu_{\mathrm{eff}}=(4.79 \pm 0.02) \mu_{\mathrm{B}}$, where $\mu_{\mathrm{B}}$ is the Bohr magneton.

The current results are very consistent with the data estimated from the $\chi(T)$ curve below $300 \mathrm{~K}$ : that is, $\Theta_{\mathrm{p}}$ 


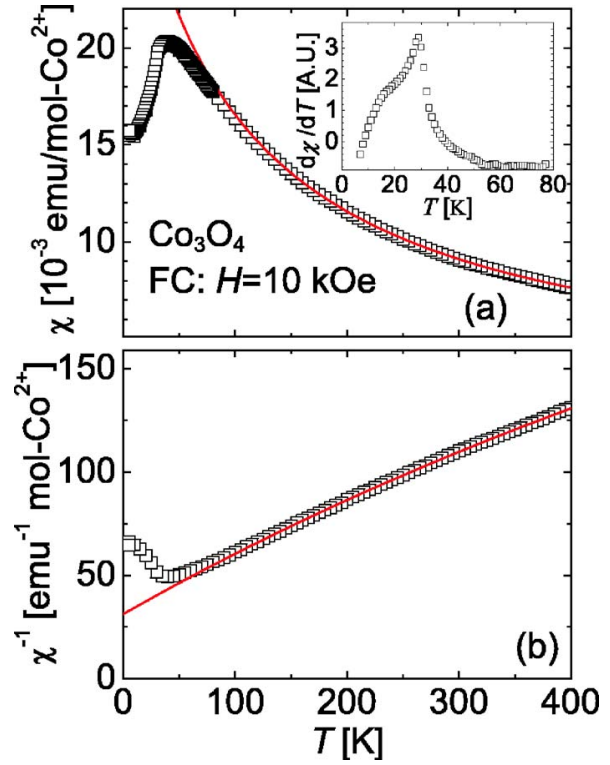

FIG. 2. (Color online) Temperature dependences of (a) magnetic susceptibility $\chi$ and (b) inverse magnetic susceptibility $\chi^{-1}$ for $\mathrm{Co}_{3} \mathrm{O}_{4}$. Solid lines represent the fit results using Eq. (1). The inset of (a) shows slope of $\chi(d \chi / d T)$ as a function of $T$.

$=108 \mathrm{~K}$ and $\mu_{\text {eff }}=4.74 \mu_{\mathrm{B}} \cdot{ }^{33}$ It should be, however, noted that Cosse reported $\Theta_{\mathrm{p}}=53 \mathrm{~K}$ and $\mu_{\mathrm{eff}}=4.14 \mu_{\mathrm{B}}$ from a $\chi$ measurement up to $1000 \mathrm{~K} .{ }^{3}$ The discrepancy between the data is probably due to the spin-state transition around $600 \mathrm{~K}$-i.e., the spin state of $\mathrm{Co}^{3+}$ ions at the $16 \mathrm{~d}$ site changes from the low- $T$ LS state $(S=0)$ to the high- $T$ HS state $(S=2) .{ }^{34} \mathrm{In}$ order to explain the nature of $\mathrm{Co}_{3} \mathrm{O}_{4}$ at low $T$, which we are primarily interested in, the data below $600 \mathrm{~K}$ are therefore considered to be suitable. In addition, $\mu_{\text {eff }}$ of the spinel $\mathrm{CoAl}_{2} \mathrm{O}_{4}\left(4.65 \mu_{\mathrm{B}}\right),{ }^{35}$ in which $\mathrm{Co}^{2+}$ ions locate only at the $8 a$ site, is in good agreement with the present result, supporting the present determination of $\Theta_{p}$ and $\mu_{\text {eff }}$ for $\mathrm{Co}_{3} \mathrm{O}_{4}$. Assuming that $\mathrm{Co}^{2+}$ ions are responsible for the Curie-Weiss paramagnetic behavior, the estimated $\mu_{\text {eff }}=4.79 \mu_{\mathrm{B}}$ is greater than the spin-only value for free $\mathrm{Co}^{2+}$ ions $\left(3.88 \mu_{\mathrm{B}}\right)$, indicating a contribution of the spin-orbit coupling of $\mathrm{Co}^{2+}$, as explained by Roth. ${ }^{4}$

Figure 3(a) shows the $T$ dependence of $C_{\mathrm{p}}$ in zero field in the $T$ range between 400 and $1.9 \mathrm{~K}$. The inset of Fig. 3(a) is a magnification of the $C_{\mathrm{p}}(T)$ curve around $30 \mathrm{~K}$. A clear $\lambda$-type peak with the maximum at $29.8 \pm 0.3 \mathrm{~K}$ in the $C_{\mathrm{p}}(T)$ curve yields the correct value of $T_{\mathrm{N}}$ for the present sample. For $\mathrm{Co}_{3} \mathrm{O}_{4}, C_{\mathrm{p}}$ has both phonon $C_{\mathrm{ph}}$ and magnetic $C_{\mathrm{m}}$ contributions. In order to estimate $C_{\mathrm{m}}$, we employ the combined Einstein-Debye model for $C_{\mathrm{ph}}$ as ${ }^{6,35}$

$$
C_{\mathrm{ph}}=9 R x_{\mathrm{D}}^{-3} \int_{0}^{x_{\mathrm{D}}} \frac{x^{4} e^{x}}{\left(e^{x}-1\right)^{2}} d x+R \sum_{i=1}^{2} a_{i} \frac{x_{\mathrm{E}_{i}}^{2} e^{x_{\mathrm{E}_{i}}}}{\left(e_{\mathrm{E}_{i}}^{x}-1\right)^{2}}
$$

The first term represents the Debye-type phonon contribution (acoustic mode) and the second term the Einstein-type contribution (two optical modes). Both $x_{\mathrm{D}}=\Theta_{\mathrm{D}} / T$ and $x_{\mathrm{E}_{i}}$ $=\Theta_{\mathrm{E}_{i}} / T$ are the reduced $T^{-1}$ for the Debye temperature $\left(\Theta_{\mathrm{D}}\right)^{i}$
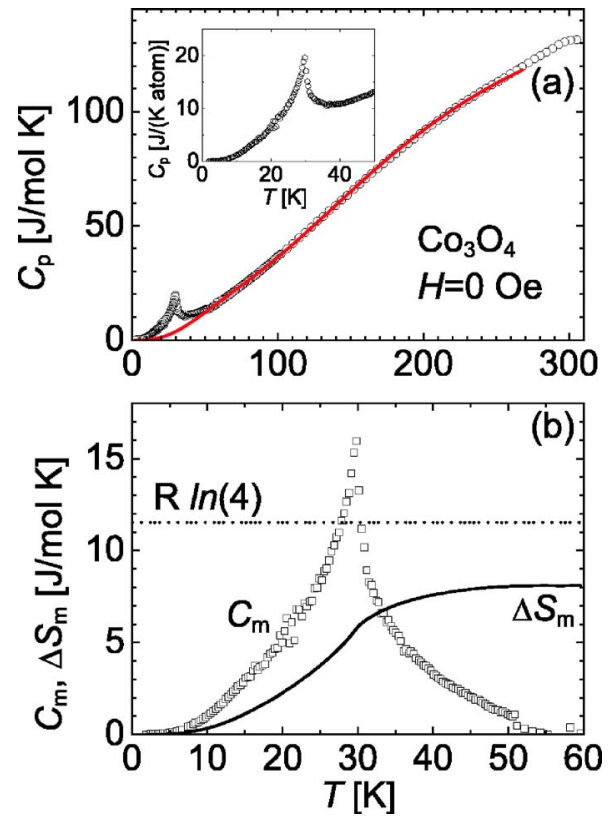

FIG. 3. (Color online) Temperature dependences of (a) $C_{\mathrm{p}}$ and (b) $C_{\mathrm{m}}$. The solid line in (a) indicates the fit result using Eq. (2) and in (b) the magnetic entropy calculated using Eq. (3). $R \ln (4)$ is the expected value of the magnetic entropy for $S=3 / 2$ system. The inset of (a) shows the magnification of the $C_{\mathrm{p}}(T)$ curve in the vicinity of $T_{\mathrm{N}}$.

and the Einstein temperature $\left(\Theta_{\mathrm{E}_{i}}\right)$, respectively. $R$ $=8.31 \mathrm{~J} /(\mathrm{mol} \mathrm{K})$ is the molar gas constant, and $a_{i}$ is the number of degree of freedom for each Einstein mode; for the cubic spinel, $a_{1}=3$ and $a_{2}=15$. $^{6}$ Above $50 \mathrm{~K}$, the $C_{\mathrm{p}}(T)$ curve is well fitted by Eq. (2), and as a result we obtain $\Theta_{\mathrm{D}}=234 \pm 3 \mathrm{~K}, \Theta_{\mathrm{E}_{1}}=311 \pm 4 \mathrm{~K}$, and $\Theta_{\mathrm{E}_{2}}=707 \pm 1 \mathrm{~K}$. Then the $C_{\mathrm{m}}(T)$ curve is calculated by subtracting $C_{\mathrm{ph}}(T)$ from the measured $C_{\mathrm{p}}(T)$ curve [see Fig. 3(b)]. The $C_{\mathrm{m}}(T)$ curve naturally exhibits a sharp maximum at $T_{\mathrm{N}}=29.8 \pm 0.3 \mathrm{~K}$. The entropy change from a magnetically ordered state to a disordered state $\left(\Delta S_{\mathrm{m}}\right)$ is calculated by the formula

$$
\Delta S_{\mathrm{m}}(T)=\int_{0}^{T} \frac{C_{\mathrm{m}}(T)}{T} d T .
$$

Figure 3(b) also shows the $\Delta S_{\mathrm{m}}(T)$ curve estimated using Eq. (3) and the magnetic entropy change expected for the $S$ $=3 / 2$ system-i.e., $R \ln (2 S+1)=11.5 \mathrm{~J} \mathrm{~K}^{-1} \mathrm{~mol}^{-1}$, while the measured $\Delta S_{\mathrm{m}}$ is estimated as $8.1 \mathrm{~J} \mathrm{~K}^{-1} \mathrm{~mol}^{-1}$ even at $60 \mathrm{~K}$. The difference is probably due to grain boundary effects in the polycrystalline sample and/or the difficulty to fit $C_{\mathrm{ph}}$, as reported in Ref. 6.

\section{B. Weak transverse field $\mu^{+} S R$}

Figure 4 shows wTF- $\mu^{+} \mathrm{SR}$ spectra for $\mathrm{Co}_{3} \mathrm{O}_{4}$ obtained at $60 \mathrm{~K}$ (squares), $30 \mathrm{~K}$ (triangles), and $8 \mathrm{~K}$ (circles) in a magnetic field of $H=50 \mathrm{Oe}$, where $\vec{H}$ is perpendicular to the initial muon spin direction $\left(\overrightarrow{S_{\mu}}\right)$. A clear muon oscillation signal due to the external field is observed at $60 \mathrm{~K}$. As $T$ 


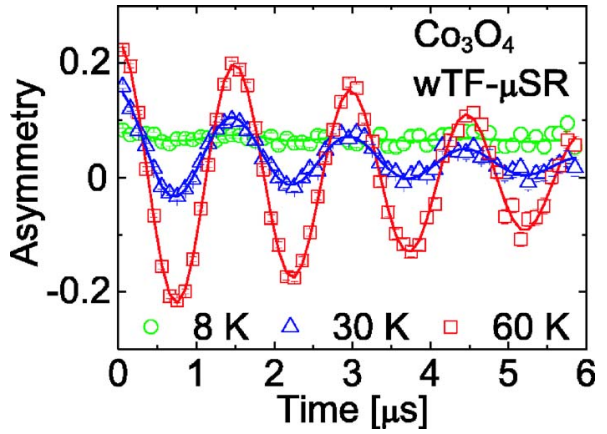

FIG. 4. (Color online) Weak transverse field (wTF-) $\mu^{+}$SR time spectra for $\mathrm{Co}_{3} \mathrm{O}_{4}$ at $60 \mathrm{~K}$ (squares), $30 \mathrm{~K}$ (triangles), and $8 \mathrm{~K}$ (circles). The magnitude of the applied wTF is 50 Oe.

decreases below $30 \mathrm{~K}$, the amplitude of this signal decreases drastically and at $8 \mathrm{~K}$ there remains only a nonoscillatory, slowly relaxing signal. Actually the wTF- $\mu^{+}$SR spectra were well fitted in the time domain using a combination of an exponentially relaxing cosine oscillation, due to the external field, and an exponentially relaxing nonoscillatory signal caused by the appearance of random internal fields in the ordered state:

$$
A_{0} P(t)=A_{\mathrm{TF}} \exp \left(-\lambda_{\mathrm{TF}} t\right) \cos \left(\omega_{\mu} t+\phi\right)+A_{\text {slow }} \exp \left(-\lambda_{\text {slow }} t\right),
$$

where $A_{0}$ is the total initial asymmetry, $P(t)$ is the muon spin polarization function, $\omega_{\mu}$ is the muon Larmor frequency, $\phi$ is the initial phase of the muon precession, and $A_{n}$ and $\lambda_{n}(n$ $=\mathrm{TF}$ and slow) are asymmetries and relaxation rates of the two signals.

Figures 5(a) and 5(b) show the $T$ dependence of $A_{n}$ and $\lambda_{n}$ for $\mathrm{Co}_{3} \mathrm{O}_{4}$ below $60 \mathrm{~K}$. Above $30 \mathrm{~K}, A_{\mathrm{TF}}$ is almost constant at its maximum value $(\sim 0.24)$, showing that the whole sample is paramagnetic. As $T$ decreases from $30 \mathrm{~K}, A_{\mathrm{TF}}$ suddenly decreases and vanishes below $29 \mathrm{~K}$, while $A_{\text {slow }}$ appears at $30 \mathrm{~K}$ and seems to level off to the constant value $(\sim 0.075)$ below $20 \mathrm{~K}$. As $T$ decreases from $60 \mathrm{~K}, \lambda_{\mathrm{TF}}$ is also

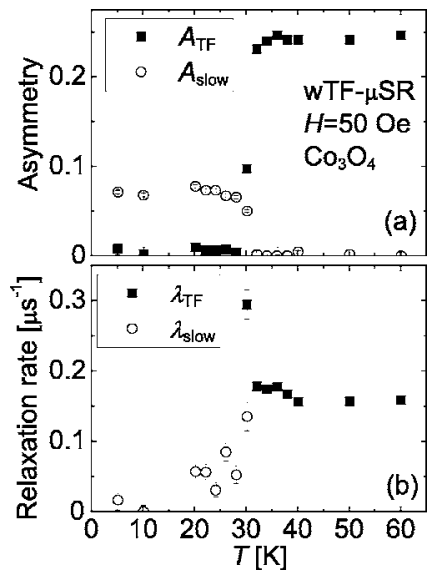

FIG. 5. Temperature dependences of (a) asymmetries $\left(A_{\mathrm{TF}}\right.$ and $\left.A_{\text {slow }}\right)$ and (b) relaxation rates $\left(\lambda_{\mathrm{TF}}\right.$ and $\left.\lambda_{\text {slow }}\right)$ for $\mathrm{Co}_{3} \mathrm{O}_{4}$. The data were obtained by fitting the wTF- $\mu^{+}$SR spectra using Eq. (4).

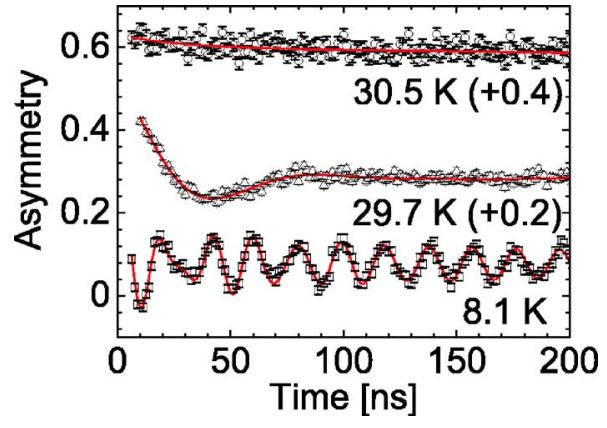

FIG. 6. (Color online) Early times part of ZF- $\mu^{+} \mathrm{SR}$ spectra for $\mathrm{Co}_{3} \mathrm{O}_{4}$ at above and below $T_{\mathrm{N}}=30 \mathrm{~K}$. The full spectra extend up to $10 \mu$ s. Solid lines represent fits using Eq. (5)

independent of $T$ down to $40 \mathrm{~K}$, then slightly increases, leaps by $0.1 \mu^{-1}$ at $30 \mathrm{~K}$, and then disappears with further lowering $T$ below $30 \mathrm{~K}$. $\lambda_{\mathrm{TF}}$ above $40 \mathrm{~K}$ is mainly due to the nuclear magnetic moments of ${ }^{59} \mathrm{Co}$ nuclei in the paramagnetic state, while the slight increase below $40 \mathrm{~K}$ would imply the formation of short-range AF order of Co ionic moments. On the other hand, $\lambda_{\text {slow }}$ decreases monotonically with decreasing $T$ below the transition, showing typical critical behavior for a transition at $T_{\mathrm{N}}$.

These indicate a sharp transition to a long-range AF order which completes below $30 \mathrm{~K}$-that is, $T_{\mathrm{N}}=30 \mathrm{~K}$, which is very consistent with the result of the $C_{\mathrm{p}}$ measurement. Moreover, $A_{\text {slow }}(5 \mathrm{~K})$ corresponds to $1 / 3$ of the maximum value of $A_{\mathrm{TF}}$, as expected for a powder sample since $1 / 3$ of the muons experience the internal field component parallel to $\overrightarrow{S_{\mu}}$ (part of the signal known as the " $1 / 3$ tail"). The remaining $2 / 3$ muon spins oscillate very rapidly due to the components of the internal field perpendicular to $\overrightarrow{S_{\mu}}$, but such oscillating signals are averaged out within the time resolution in Fig. 4 and do not contribute to the signal at longer times. Since $A_{\mathrm{TF}}$ is proportional to the volume fraction of paramagnetic phases in the sample, the wTF results show that the whole sample volume enters into the AF phase.

\section{Zero-field $\mu^{+} \mathbf{R S}$}

In order to investigate the internal magnetic field $\left(\vec{H}_{\text {int }}\right)$ of the AF phase for $\mathrm{Co}_{3} \mathrm{O}_{4}$ in detail, $\mathrm{ZF}-\mu^{+} \mathrm{SR}$ experiments were performed below $31 \mathrm{~K}$. Figure 6 shows $\mathrm{ZF}-\mu^{+} \mathrm{SR}$ time spectra at $30.5 \mathrm{~K}$ (top), $29.7 \mathrm{~K}$ (middle), and $8.1 \mathrm{~K}$ (bottom). A clear spontaneous muon spin oscillatory signal due to a quasistatic $\vec{H}_{\text {int }}$ is observed below $29.7 \mathrm{~K}$ ("quasistatic" means static at least within the time scale of $\mu^{+} \mathrm{SR}-$-i.e. up to $\sim 10 \mu \mathrm{s}$ ), whereas there is no oscillation signal at $30.5 \mathrm{~K}$, further indicating the appearance of a long-range $\mathrm{AF}$-ordered state below $29.7 \mathrm{~K}$.

The Fourier transforms of ZF- $\mu^{+}$SR time spectra clearly show two distinct frequency components in each spectrum (see Fig. 7). The spectrum at $8 \mathrm{~K}$ consists of a main peak at $\nu_{2}=51.6 \mathrm{MHz}$ and a second broad weaker maximum at $\nu_{1}$ $=72.7 \mathrm{MHz}$, corresponding to $H_{\mathrm{int}, 2}=3.8 \mathrm{kOe}$ and $H_{\mathrm{int}, 1}$ $=5.4 \mathrm{kOe}$, respectively. Both $\nu_{1}$ and $\nu_{2}$ monotonically de- 


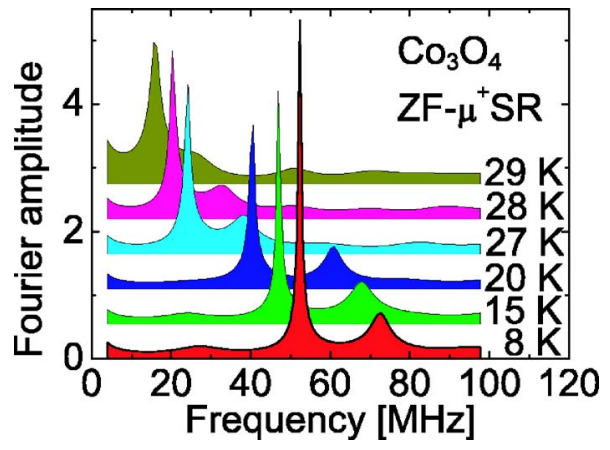

FIG. 7. (Color online) Fourier amplitude of ZF- $\mu^{+}$SR spectra at several temperatures below $T_{\mathrm{N}}$.

crease with increasing $T$ and disappear simultaneously at $30 \mathrm{~K}$.

The full widths at half maximum (FWHM) of the two peaks at $\nu_{1}$ and $\nu_{2}$ increase with increasing $T$, and the former is broader than the latter in the whole $T$ range measured. In the layered cobalt oxides, such as $\mathrm{Na}_{x} \mathrm{CoO}_{2}$ and $\left[\mathrm{Ca}_{2} \mathrm{CoO}_{3}\right]_{0.62}^{\mathrm{RS}}\left[\mathrm{CoO}_{2}\right]$, coexisting $\mathrm{Co}^{3+}$ and $\mathrm{Co}^{4+}$ ions in the $\mathrm{CoO}_{2}$ planes naturally induce the wide distribution of $H_{\text {int }}$. On the contrary, in the $\mathrm{Co}_{3} \mathrm{O}_{4}$ spinel, $\mathrm{Co}^{2+}$ ions locate only at the $8 a$ site. As a result, the FWHM $(T=8 \mathrm{~K})$ at $\nu_{1}$ is $6.0 \mathrm{MHz}$ and at $\nu_{2} 1.6 \mathrm{MHz}$, respectively, while the FWHM of the main peak $(\nu=55 \mathrm{MHz})$ for $\left[\mathrm{Ca}_{2} \mathrm{CoO}_{3}\right]_{0.62}^{\mathrm{RS}}\left[\mathrm{CoO}_{2}\right]$ is about $20 \mathrm{MHz}$ at $2.5 \mathrm{~K}$ and for $\mathrm{Na}_{0.75} \mathrm{CoO}_{2} 1 \mathrm{MHz}(\nu$ $=3.3 \mathrm{MHz}$ ) at $2.5 \mathrm{~K}$. This shows that the distribution of $H_{\text {int }}$ in $\mathrm{Co}_{3} \mathrm{O}_{4}$ is rather small compared with those in the layered cobalt oxides, as expected.

There are two possible reasons for the appearance of the two oscillatory components in the ZF time spectra: namely, the presence of two magnetically inequivalent $\mu^{+}$sites and an incommensurate (IC) magnetic order in the AF phase of $\mathrm{Co}_{3} \mathrm{O}_{4}$. In the former case, a phenomenological relaxation function for the oscillatory components in the ZF time spectrum is a combination of two exponentially damped cosine signals, $\sum A_{n} \exp \left(-\lambda_{n} t\right) \cos \left(2 \pi \nu_{n} t+\phi_{n}\right)\left(n=1\right.$ and $\left.2, \nu_{1}>\nu_{2}\right)$. Actually, the ZF time spectra for $\mathrm{Co}_{3} \mathrm{O}_{4}$ below $T_{\mathrm{N}}$ are well fitted by this phenomenological function except that the fit results exhibit a large negative phase shift for both oscillatory signals; i.e., the delay of the initial phases are $\phi_{1}=$ $-70^{\circ} \pm 5^{\circ}$ and $\phi_{2}=-48^{\circ} \pm 4^{\circ}$ at $8 \mathrm{~K}$. This indicates the appearance of the IC magnetic order below $T_{\mathrm{N}}$ for $\mathrm{Co}_{3} \mathrm{O}_{4}$, because $\phi$ should be zero for commensurate (C-)AF order. ${ }^{36}$ Furthermore, as $T$ decreases from $30 \mathrm{~K}, A_{1}\left(A_{2}\right)$ increases (decreases) monotonically with $T$ from $0.06(0.10)$ at $30 \mathrm{~K}$ to $0.11(0.06)$ at $8 \mathrm{~K}$. In order to explain the $A_{1}(T)$ and $A_{2}(T)$ curves, one would have to assume the existence of two muon sites and a situation whereby the population of $\mu^{+}$at each site changes monotonically with $T$. Such behavior is very unlikely to occur at low $T$.

The ZF time spectra were therefore fitted with the following formula: ${ }^{37}$

$$
\begin{aligned}
A_{0} P(t)= & A_{1} J_{0}\left(2 \pi \nu_{1} t\right) \exp \left(-\lambda_{1} t\right)+A_{2} \exp \left(-\lambda_{2} t\right) \\
& \times \cos \left(2 \pi \nu_{2} t+\phi\right)+A_{\text {slow } 1} \exp \left(-\lambda_{\text {slow } 1} t\right) \\
& +A_{\text {slow 2 }} \exp \left(-\lambda_{\text {slow } 2} t\right)
\end{aligned}
$$

$$
\nu_{1}>\nu_{2}
$$

where $A_{0}$ is the total initial asymmetry, $P(t)$ is the muon polarization function, $A_{n}, \lambda_{n}$, and $\nu_{n}(n=1$ and 2) are the asymmetries, relaxation rates, and oscillation frequencies of the two oscillating signals, respectively, $\phi$ is the initial phase of the exponentially damped term, and $A_{\text {slow }, n}$ and $\lambda_{\text {slow, } n}(n$ $=1$ and 2) are the asymmetries and relaxation rates of the non-oscillating signals, respectively. $J_{0}\left(2 \pi \nu_{1} t\right)$ is the zerothorder Bessel function of the first kind. This is because according to the lattice sum calculation, a generalized IC field distribution at the muon site $H$ is given by ${ }^{30,37,38}$

$$
\begin{gathered}
P(H)=\frac{2}{\pi} \frac{H}{\sqrt{\left(H_{1}^{2}-H^{2}\right)\left(H^{2}-H_{2}^{2}\right)}}, \\
H_{2}<H<H_{1},
\end{gathered}
$$

where $2 \pi \nu_{1}=\gamma_{\mu} H_{1}$ and $2 \pi \nu_{2}=\gamma_{\mu} H_{2}\left(\gamma_{\mu}\right.$ is the muon gyromagnetic ratio). Since Eq. (6) represents the field distribution in a frequency domain, Eq. (6) is unavailable to fit $\mathrm{ZF}-\mu^{+} \mathrm{SR}$ time spectra. On the other hand, the sum of $J_{0}(2 \pi \nu t)$ and the cosine function, which corresponds to the first two terms in Eq. (5), is known to well represent the fine spectrum of $P(H)$. We thus used Eq. (5) to fit the ZF- $\mu^{+} \mathrm{SR}$ spectra of $\mathrm{Co}_{3} \mathrm{O}_{4}$. The two nonoscillating components in Eq. (5) correspond to the " $1 / 3$ tail," as described in the previous section on wTF- $\mu^{+} \mathrm{SR}$ results.

Figure 8 shows the $T$ dependences of (a) $\lambda_{n}$, (b) $A_{1}+A_{2}$ and $A_{\text {slow } 1}+A_{\text {slow2 }}$, and (c) $\nu_{n}$ and $\Delta \nu=\nu_{1}-\nu_{2}$ of the two oscillating signals $(n=1$ and 2$)$ obtained by the fitting results using Eq. (5). As $T$ decreases from $30 \mathrm{~K}$, both $\lambda_{1}$ and $\lambda_{2}$ rapidly decrease with decreasing slope and reach their minima below $\sim 15 \mathrm{~K}$. This is reasonably explained by the critical phenomenon in the magnetically ordered system at the vicinity of $T_{\mathrm{N}}$.

$A_{1}+A_{2}$ is almost $T$ independent below $T_{\mathrm{N}}$ and levels off to its maximum value $(\sim 0.15)$ for a magnetic powder sample. $A_{\text {slow1 }}+A_{\text {slow2 }}$ is also $T$ independent below $T_{\mathrm{N}}$ and levels off to half the value of $A_{1}+A_{2}(\sim 0.08)$. This indicates that $A_{\text {slow1 }}+A_{\text {slow2 }}$ represent the " $1 / 3$ tail," which is consistent with the result of wTF- $\mu \mathrm{SR}$. The result is further evidence supporting the conjecture that the whole sample enters into the IC-AF order phase below $T_{\mathrm{N}}$.

The two $\nu_{n}(T)$ curves exhibit a similar $T$ dependence [see Fig. 8(c)]. As $T$ decreases from $31 \mathrm{~K}$, both $\nu_{1}$ and $\nu_{2}$ suddenly appear at $30 \mathrm{~K}$ and increase with decreasing $T$, with decreasing slope. At the vicinity of $T_{\mathrm{N}}, \Delta \nu$ rapidly increases with decreasing $T$ and seems to level off to $\sim 20 \mathrm{MHz}$ below $20 \mathrm{~K}$. Since $\Delta \nu$ measures the field distribution of the IC order, this implies that the IC AF structure of $\mathrm{Co}_{3} \mathrm{O}_{4}$ is completed at $20 \mathrm{~K}$. This is consistent with the result of the $T$ dependences of the $\lambda_{n}$ as described above.

The other parameters $A_{\text {slow1 }}, A_{\text {slow2 }}, \lambda_{\text {slow1 }}$, and $\lambda_{\text {slow2 }}$ lack $T$ dependence below $T_{\mathrm{N}}$, indicating that the implanted muon does not change the site at least in the time scale of the muon lifetime. The $\phi(T)$ curve is independent of $T$ with $\phi \sim 10^{\circ}$ at 


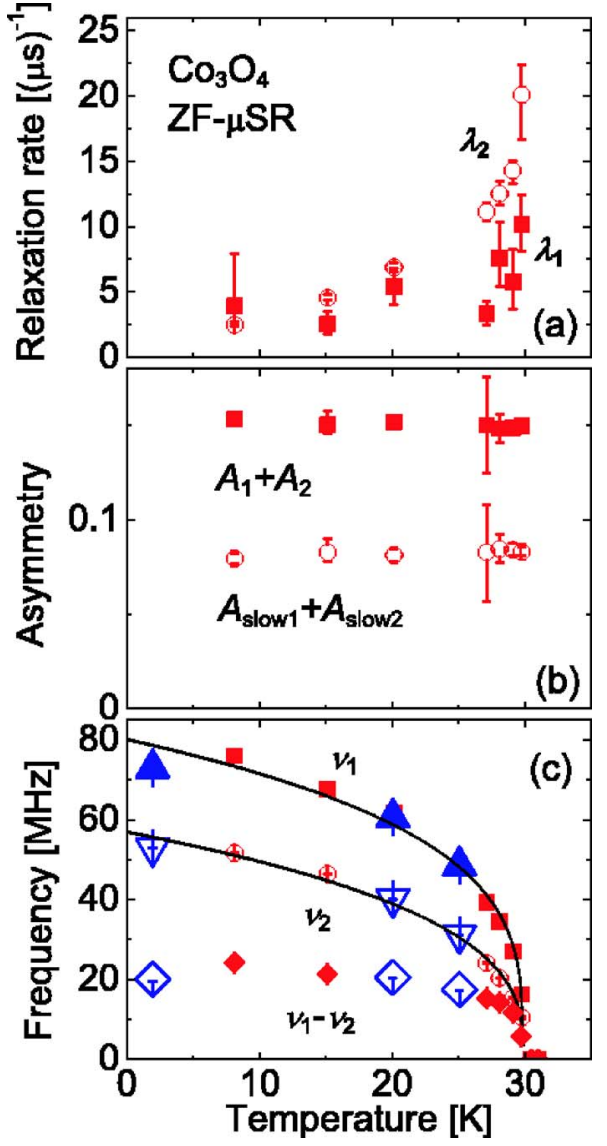

FIG. 8. (Color online) Temperature dependences of (a) $\lambda_{n}$, (b) $A_{1}+A_{2}$ and $A_{\text {slow } 1}+A_{\text {slow2 }}$, and (c) $\nu_{n}\left(n=1\right.$ and 2) and $\Delta \nu=\nu_{1}-\nu_{2}$. These results were obtained by fitting the ZF- $\mu^{+}$SR spectra with Eq. (5). Solid curves are guide to the eye. $\boldsymbol{\Delta}, \nabla, \diamond$ and data obtained at TRIUMF and $\boldsymbol{\square}, \bigcirc$, and $\bullet$ and at PSI.

whole temperatures measured. This phase shift is due to the rotation of the initial muon spin direction by $10^{\circ}$ to avoid direct positrons from the muon source.

\section{DISCUSSION}

\section{A. Origin of incommensurate order}

Here, we discuss the origin of the IC-AF order in $\mathrm{Co}_{3} \mathrm{O}_{4}$. To begin with, the magnetic interaction between $\mathrm{Co}^{2+}$ ions at the $8 a$ site is known to proceed via multiple exchange paths involving several $\mathrm{Co}^{2+}-\mathrm{O}-\mathrm{Co}^{3+}-\mathrm{O}-\mathrm{Co}^{2+}$ bonds (see Fig. 1). ${ }^{4}$ Additionally, among related materials, $\mathrm{CoAl}_{2} \mathrm{O}_{4}$ lacks a magnetically ordered state even at $4 \mathrm{~K},{ }^{39}$ while $\mathrm{CoRh}_{2} \mathrm{O}_{4}$, in which $\mathrm{Rh}^{3+}$ is in the $4 d^{6}$ configuration of LS state $(S=0)$, exhibits a long-range AF order below $T_{\mathrm{N}}=27 \mathrm{~K} .{ }^{40}$ This suggests that the vacant $e_{g}$ orbitals at the $16 d$ site play an important role in the AF order in $\mathrm{Co}_{3} \mathrm{O}_{4}$. We therefore need to take into account not only the nature of the magnetic $\mathrm{Co}^{2+}$ ions at the $8 a$ site but also the charge and/or spin fluctuations of $\mathrm{Co}^{3+}$ at the $16 d$ site for explaining the origin of the IC-AF order.

Since the exchange path through three intervening ions produces a relatively weak superexchange interaction, which is responsible for the $\mathrm{AF}$ ordering of $\mathrm{Co}_{3} \mathrm{O}_{4}$, either a slight local structural distortion around $\mathrm{Co}$ ions or the change in charge and spin of $\mathrm{Co}^{3+}$ would alter the overlap between the orbitals of $\mathrm{Co}^{3+}$ and $\mathrm{O}^{2-}$, resulting in a modulation of the local internal magnetic field of $\mathrm{Co}_{3} \mathrm{O}_{4}$.

According to a recent neutron diffraction study for a cubic spinel $\mathrm{MnSc}_{2} \mathrm{~S}_{4}=\left[\mathrm{Mn}^{2+}\right]_{8 a}\left[\mathrm{Sc}_{2}^{3+}\right]_{16 d}\left[\mathrm{~S}_{4}^{2-}\right]_{32 e}$ with $T_{N}=2.3 \mathrm{~K}^{41}$ an IC-SDW order with a spiral magnetic structure was found below $T_{\mathrm{N}}$, although there are no structural phase transitions down to $1.5 \mathrm{~K}$. The origin of the IC-SDW order in $\mathrm{MnSc}_{2} \mathrm{~S}_{4}$ was proposed as a local structural transition due to spindriven Jahn-Teller (JT) effects below $T_{\mathrm{N}}$. Such a transition was, however, considered to be undetectable, because the distortion is probably far beyond the resolution of the current neutron diffraction technique.

A similar scenario would be applicable for $\mathrm{Co}_{3} \mathrm{O}_{4}$, which also keeps a cubic symmetry down to $4.2 \mathrm{~K},{ }^{4}$ if Co ions are JT active. The possible reactions in the AF phase of $\mathrm{Co}_{3} \mathrm{O}_{4}$ are therefore as follows.

(i) Spin-state transition of $\mathrm{Co}^{3+}$ ions at the $16 d$ site: $\mathrm{Co}^{3+}(\mathrm{LS}, S=0) \rightarrow \mathrm{Co}^{3+}$ (HS, $S=1$ or $S=2$ ).

(ii) Charge disproportionation of $\mathrm{Co}^{3+}$ ions at the $16 d$ site into $\mathrm{Co}^{2+}$ and $\mathrm{Co}^{4+}$ ions: $\mathrm{Co}^{3+}(\mathrm{LS}) \rightarrow \mathrm{Co}^{2+}(\mathrm{HS}, \mathrm{S}=3 / 2)$ $+\mathrm{Co}^{4+}(S=1 / 2)$.

(iii) Exchange of $\mathrm{Co}^{2+}$ ion at the $8 a$ site with $\mathrm{Co}^{3+}$ ion at the $16 d$ site: $\mathrm{Co}^{2+}(8 a, S=3 / 2)+\mathrm{Co}^{3+}(16 d, S=0) \rightarrow \mathrm{Co}^{3+}$ $(8 a, S=2)+\mathrm{Co}^{2+}(16 d, S=3 / 2)$.

All the reactions listed above are assigned to an electron transfer process between Co ions with activation energies ranging from 0.3 to $0.7 \mathrm{eV} .{ }^{34}$ The above reactions are thus likely to occur locally even at low $T$ and, as a result, induce a local JT distortion in the $\mathrm{CoO}_{6}$ octahedra. Such distortion naturally leads to a fluctuation of the distance between $\mathrm{Co}^{2+}$ ions and $\mu^{+}$. We should here note that, although there is no evidence for IC-SDW order in the past neutron powder diffraction measurements, it is not impossible but very difficult to detect the IC-SDW order by neutron. This is because the IC modulation reduces the intensity of magnetic Bragg peaks. In order to further understanding of the origin of the inhomogeneity, additional $\mu^{+} \mathrm{SR}$ experiments are required using single-crystal samples of $\mathrm{Co}_{3} \mathrm{O}_{4}$ to determine the $\mu^{+}$ sites more precisely and to know the relationship between $H_{\text {int }}$ and the easy axis of magnetization.

\section{B. Comparison with layered cobalt oxides}

In earlier $\mu^{+} \mathrm{SR}$ work, two major precessing signals with $\nu_{n}(0 \mathrm{~K})=54$ and $78 \mathrm{MHz}$, plus a minor signal at $150 \mathrm{MHz}$, were observed in a rocksalt-type $\mathrm{CoO}\left(\mathrm{Co}^{2+} \mathrm{O}^{2-}\right)$ crystal, ${ }^{26}$ comparable to the present experimental values of $\nu_{n}(0 \mathrm{~K})$ $=60$ and $80 \mathrm{MHz}$ for the $\left[\mathrm{Co}^{2+}\right]_{8 a}\left[\mathrm{Co}_{2}^{3+}\right]_{16 d}\left[\mathrm{O}_{4}^{2-}\right]_{32 e}$ spinel. In the AF phase of $\mathrm{CoO}$ below $T_{\mathrm{N}}=290 \mathrm{~K}$, the spins of $\mathrm{Co}^{2+}$ ions align parallel to each other on (111) planes, but with antiparallel spin directions in alternate (111) planes. It is therefore very surprising that $H_{\text {int }}$ detected by $\mu^{+}$for $\mathrm{CoO}$ are almost the same as for $\mathrm{Co}_{3} \mathrm{O}_{4}$, in spite of the fact that the magnetic structure of $\mathrm{CoO}$ is so different from that of $\mathrm{Co}_{3} \mathrm{O}_{4}$. This could suggest the existence of $\mathrm{Co}^{2+}$ ions in the $16 d$ site in $\mathrm{Co}_{3} \mathrm{O}_{4}$, as in the case for the well-known $\mathrm{Fe}_{3} \mathrm{O}_{4}$ 
$=\left[\mathrm{Fe}^{3+}\right]_{8 a}\left[\mathrm{Fe}^{2+} \mathrm{Fe}^{3+}\right]_{16 d}\left[\mathrm{O}_{4}^{2-}\right]_{32 e}$ case. However, $\mathrm{Co}^{2+}$ ions are most unlikely to exist in $\left[\mathrm{Ca}_{2} \mathrm{CoO}_{3}\right]_{0.62}^{\mathrm{RS}}\left[\mathrm{CoO}_{2}\right]$ based on $\chi$ measurements, ${ }^{42}$ a bond-valence-sum calculation using the structural refinement data, ${ }^{43}$ and a photoelectron spectroscopic analysis at ambient temperature. ${ }^{44}$ Nevertheless, assuming that a charge disproportionation of $\mathrm{Co}^{3+}$ ions $\left(2 \mathrm{Co}^{3+} \rightarrow \mathrm{Co}^{2+}+\mathrm{Co}^{4+}\right)$ occurs in the $\mathrm{CoO}_{2}$ planes at low $T$ as in the case for $\mathrm{LiCoO}_{2}, 22$ it would be reasonable that the IC-SDW with $\nu(0 \mathrm{~K})=54 \mathrm{MHz}$ exists in the $\mathrm{CoO}_{2}$ planes in $\left[\mathrm{Ca}_{2} \mathrm{CoO}_{3}\right]_{0.62}^{\mathrm{RS}}\left[\mathrm{CoO}_{2}\right]$. Although the current $\mu^{+} \mathrm{SR}$ results on $\mathrm{Co}_{3} \mathrm{O}_{4}$ do not provide crucial information concerning the position of the IC-SDW in the layered cobalt oxides, indirect evidence, if $\mathrm{Co}^{2+}$ ions exist in the $16 d$ octahedral site, still seems to support that the IC-SDW lies in the $\mathrm{CoO}_{2}$ planes.

\section{SUMMARY}

The magnetic properties of $\mathrm{Co}_{3} \mathrm{O}_{4}$ below $T_{\mathrm{N}}$ were studied in wTF- and ZF- $\mu^{+}$SR experiments, together with magnetic susceptibility and heat capacity measurements. Spontaneous muon spin precession signals with two different frequencies $77 \pm 4$ and $56 \pm 3 \mathrm{MHz}(T \rightarrow 0)$ were observed in $\mathrm{ZF}-\mu^{+} \mathrm{SR}$ spectra below $30 \mathrm{~K}$, both with sizable inhomogeneous broadening. The precise analyses of the ZF spectra also suggested the formation of an incommensurate antiferromagnetic order below $T_{\mathrm{N}}$. Although the origin of the inhomogeneity of the magnetic moment in the AF order state is not fully understood, it could be caused by a slight structural distortion together with a spin and/or charge state transition of $\mathrm{Co}^{3+}$ ions at the $16 d$ site. Experiments on not only a single crystal $\mathrm{Co}_{3} \mathrm{O}_{4}$ but also related materials, such as, $\mathrm{CoRh}_{2} \mathrm{O}_{4}$, $\mathrm{ZnCo}_{2} \mathrm{O}_{4}$ (diamagnetic ions at $8 a$ sites), and $\mathrm{CoAl}_{2} \mathrm{O}_{4}$ (diamagnetic ions at $16 d$ sites), help to elucidate the role of $\mathrm{Co}^{3+}$ ions at the $16 d$ site on the long-range AF order.

\section{ACKNOWLEDGMENTS}

This work was performed at both TRIUMF, Vancouver, Canada and the Swiss Muon Source, Paul Sherrer Institut, Villigen, Switzerland. We thank S. R. Kreitzmann, B. Hitti, and D. J. Arseneau of TRIUMF, P. Russo of Columbia University, and LMU staff of PSI for help with the $\mu^{+}$SR experiments. This work was partially (J.H.B.) supported at UBC by CIAR, NSERC of Canada, and at TRIUMF by NRC of Canada.
${ }^{1}$ K. Miyatani, K. Kohn, H. Kamimura, and S. Iida, J. Phys. Soc. Jpn. 21, 464 (1966).

${ }^{2}$ H. Kamimura, J. Phys. Soc. Jpn. 21, 484 (1966).

${ }^{3}$ P. Cosse, J. Inorg. Nucl. Chem. 8, 483 (1958).

${ }^{4}$ W. L. Roth, J. Phys. Chem. Solids 25, 1 (1964).

${ }^{5}$ W. Kundig, M. Kobelt, H. Appel, G. Constabaris, and R. H. Lindquist, J. Phys. Chem. Solids 30, 819 (1969).

${ }^{6}$ L. M. Khriplovich, E. V. Kholopov, and I. E. Paukov, J. Chem. Thermodyn. 14, 207 (1982).

${ }^{7}$ Y. Ichiyanagi, Y. Kimishima, and S. Yamada, J. Magn. Magn. Mater. 272-276, e1245 (2004).

${ }^{8}$ B. Pejova, A. Isahi, M. Najdoski, and I. Grozdanov, Mater. Res. Bull. 36, 161 (2001).

${ }^{9}$ C. Nethravathi, S. Sen, N. Ravishankar, M. Rajamathi, C. Pietzonka, and B. Harbrecht, J. Phys. Chem. B 109, 11468 (2005).

${ }^{10}$ W. L. Smith and A. D. Hobson, Acta Crystallogr., Sect. B: Struct. Crystallogr. Cryst. Chem. 29, 362 (1973).

${ }^{11}$ J. Molenda, C. Delmas, P. Dordor, and A. Stoklosa, Solid State Ionics 12, 473 (1989).

${ }^{12}$ R. Funahashi, I. Matsubara, H. Ikuta, T. Takenouchi, U. Mizukami, and S. Sodeoka, Jpn. J. Appl. Phys., Part 2 39, L1127 (2000).

${ }^{13}$ Y. Miyazaki, T. Miura, Y. Ono, and T. Kajitani, Jpn. J. Appl. Phys., Part 2 41, L849 (2002).

${ }^{14}$ J. Sugiyama, J. H. Brewer, E. J. Ansaldo, H. Itahara, T. Tani, M. Mikami, Y. Mori, T. Sasaki, S. Hebert, and A. Maignan, Phys. Rev. Lett. 92, 017602 (2004).

${ }^{15}$ J. Sugiyama, H. Itahara, J. H. Brewer, E. J. Ansaldo, T. Motohashi, M. Karppinen, and H. Yamauchi, Phys. Rev. B 67, 214420 (2003).

${ }^{16}$ J. Sugiyama, J. H. Brewer, E. J. Ansaldo, B. Hitti, M. Mikami, Y. Mori, and T. Sasaki, Phys. Rev. B 69, 214423 (2004).
${ }^{17}$ J. Sugiyama, H. Itahara, T. Tani, J. H. Brewer, and E. J. Ansaldo, Phys. Rev. B 66, 134413 (2002).

${ }^{18}$ J. Sugiyama, J. H. Brewer, E. J. Ansaldo, H. Itahara, K. Dohmae, Y. Seno, C. Xia, and T. Tani, Phys. Rev. B 68, 134423 (2003).

${ }^{19}$ J. Sugiyama, H. Nozaki, J. H. Brewer, E. J. Ansaldo, T. Takami, H. Ikuta, and U. Mizutani, Phys. Rev. B 72, 064418 (2005).

${ }^{20}$ J. Sugiyama, J. H. Brewer, E. J. Ansaldo, H. Itahara, K. Dohmae, C. Xia, Y. Seno, B. Hitti, and T. Tani, J. Phys.: Condens. Matter 15, 8619 (2003).

${ }^{21}$ J. Sugiyama, H. Nozaki, Y. Ikedo, K. Mukai, J. H. Brewer, E. J. Ansaldo, G. D. Morris, D. Andreica, A. Amato, T. Fujii, and A. Asamitsu, Phys. Rev. Lett. 96, 037206 (2006).

${ }^{22}$ J. Sugiyama, H. Nozaki, J. H. Brewer, E. J. Ansaldo, G. D. Morris, and C. Delmas, Phys. Rev. B 72, 144424 (2005).

${ }^{23}$ L. M. Helme, A. T. Boothroyd, R. Coldea, D. Prabhakaran, D. A. Tennant, A. Hiess, and J. Kulda, Phys. Rev. Lett. 94, 157206 (2005)

${ }^{24}$ S. P. Bayrakci, I. Mirebeau, P. Bourges, Y. Sidis, M. Enderle, J. Mesot, D. P. Chen, C. T. Lin, and B. Keimer, Phys. Rev. Lett. 94, 157205 (2005).

${ }^{25}$ J. Sugiyama (unpublished).

${ }^{26}$ K. Nishiyama, S. Ohira, W. K. Dawson, and W. Higemoto, Hyperfine Interact. 104, 349 (1997).

${ }^{27}$ A. C. Masset, C. Michel, A. Maignan, M. Hervieu, O. Toulemonde, F. Studer, B. Raveau, and J. Hejtmanek, Phys. Rev. B 62, 166 (2000)

${ }^{28}$ Y. J. Uemura, W. J. Kossler, X. H. Yu, J. R. Kempton, H. E. Schone, D. Opie, C. E. Stronach, D. C. Johnston, M. S. Alvarez, and D. P. Goshorn, Phys. Rev. Lett. 59, 1045 (1987).

${ }^{29}$ L. P. Le et al., Phys. Rev. B 53, R510 (1996).

${ }^{30}$ G. M. Kalvius, D. R. Noakes, and O. Hartmann, Handbook on the Physics and Chemistry of Rare Earths (North-Holland, Amster- 
dam, 2001), Vol. 32.

${ }^{31}$ A. T. Savici et al., Phys. Rev. B 66, 014524 (2002).

${ }^{32}$ Y. J. Uemura, Muon Science (Institute of Physics, Bristol, 1999).

${ }^{33}$ T. Fukai, Y. Furukawa, S. Wada, and K. Miyatani, J. Phys. Soc. Jpn. 63, 4067 (1996).

${ }^{34}$ V. A. M. Brabers and A. D. D. Broemme, J. Magn. Magn. Mater. 104-107, 405 (1992).

${ }^{35}$ N. Tristan, J. Hemberger, A. Krimmel, J.-A. Krug von Nidda, V. Tsurkan, and A. Loidl, Phys. Rev. B 72, 174404 (2005).

${ }^{36}$ K. M. Kojima et al., Phys. Rev. Lett. 79, 503 (1997).

${ }^{37}$ J. Sugiyama, Y. Ikedo, K. Mukai, J. H. Brewer, E. J. Ansaldo, G. D. Morris, K. H. Chow, H. Yoshida, and Z. Hiroi, Phys. Rev. B
73, 224437 (2006).

${ }^{38}$ D. A. Andreica, Ph.D. thesis, ETH-Zurich, 2001.

${ }^{39}$ W. L. Roth, J. Phys. (Paris) 25, 507 (1964).

${ }^{40}$ G. Blasse, Phys. Lett. 19, 110 (1965).

${ }^{41}$ A. Krimmel, M. Mücksch, V. Tsurkan, M. M. Koza, H. Mutka, C. Ritter, D. V. Sheptyakov, S. Horn, and A. Loidl, Phys. Rev. B 73, 014413 (2006).

${ }^{42}$ J. Sugiyama, C. Xia, and T. Tani, Phys. Rev. B 67, 104410 (2003).

${ }^{43}$ Y. Miyazaki, M. Onoda, T. Oku, M. Kikuchi, Y. Ishii, Y. Ono, Y. Morii, and T. Kajitani, J. Phys. Soc. Jpn. 71, 491 (2002).

${ }^{44}$ Y. Miyazaki (private communication). 